\title{
A Universal Correlation Predicts Permeability Coefficients of Fluid- and Gel- Phase Phospholipid and Phospholipid-Cholesterol Bilayers for Arbitrary Solutes
}

\author{
Johannes M. Nitsche ${ }^{1}$ and Gerald B. Kasting ${ }^{2}$ \\ ${ }^{1}$ Department of Chemical and Biological Engineering \\ University at Buffalo, The State University of New York \\ Buffalo, NY 14260-4200 \\ ${ }^{2}$ James L. Winkle College of Pharmacy \\ University of Cincinnati Academic Health Center \\ Cincinnati, Ohio 45267-0004
}

Keywords: permeability; bilayer; phospholipids; passive diffusion/transport; membrane transport; mathematical model; bioavailability 


\begin{abstract}
The permeability of gel-phase phospholipids is typically about an order of magnitude lower than that of the same compositions in the fluid phase, yet a quantitative description of the ordering factors leading to this difference has been elusive. The present analysis examines these factors with particular focus on the area per phospholipid chain, $A_{\mathrm{c}}$, and its relationship to the minimum area per molecule in the crystalline state, $A_{0}$. It is shown that fluid- and gel-phase phospholipid permeabilities can be reconciled by postulating a minimum area per chain $A_{\mathrm{c}, 0}=17.1 \AA^{2}$, substantially less than one would estimate by dividing the accepted value $A_{0}=40.8 \AA^{2}$ by two. An extended data set of phospholipid and phospholipid-cholesterol bilayer permeability data extending over 9 orders of magnitude is analyzed and correlated according to the developed relationship $\left(N=85, s=0.3024, r^{2}=0.9332\right)$. Individual permeability values are consequently predicted to within an average deviation of $10^{0.3024}$, or about a factor of two. The analysis is broadly applicable in the fluid phase, but is restricted to gel-phase phospholipid compositions that do not contain cholesterol. Guidance for the latter scenario is provided.
\end{abstract}




\section{INTRODUCTION}

This paper addresses the prediction of the permeability coefficient $P^{\text {lip/w }}$ of a phospholipid or phospholipid-cholesterol bilayer as a function of the molecular structure and properties of the permeating solute (molecular weight $\mathrm{MW}$, octanol/water partition coefficient $K^{\text {oct } / \text { ww }}$, etc.). The lipids considered (egg lecithin phosphatidylcholines (EPC), dioleoylphosphatidylcholine (DOPC), dimyristoylphosphatidylcholine (DMPC), dipalmitoylphosphatidylcholine (DPPC) and distearoylphosphatidylcholine (DSPC)) are broadly representative of the phospholipid components of cell plasma membranes. This fact makes the correlation for $P^{\text {lip/w }}$ developed directly relevant to the quantitative assessment of molecular bioavailability in such crucial applications as absorption-distribution-metabolism-excretion (ADME) screening of drug candidates, ${ }^{1,2}$ and permeability profiling in particular, ${ }^{3}$ as well as risk assessment of chemical exposures through the skin ${ }^{4-6}$ and other pathways. ${ }^{6}$ Through the inclusion of more fluid-phase data, and more crucially also gel-phase data, this paper represents a substantial generalization of a previous analysis of fluid-phase bilayer permeability, ${ }^{7}$ henceforth referred to as Part I.

The analysis is based upon an extensive body of experimental work from the laboratory of Anderson, Xiang and coworkers, and it builds upon important mechanistic deductions made by these authors. The pharmacological literature has seen the devotion of considerable effort ${ }^{7-10}$ to the development of correlations for $P^{\text {lip/w }}$ because - if reliable - theoretical formulas represent an attractive alternative to experimental assays, which could be carried out for at best a small fraction of the molecules represented in typical lists of drug candidates or chemicals to which the population is exposed. The present analysis constructs a mechanistic description of chain ordering effects as a superstructure upon the backbone of an appropriate measure of lipophilicity of the permeating solute. Within this framework, specification of permeant size and 
lipid density is sufficient to: (i) describe fluid-phase permeabilities at a level of accuracy comparable to or better than that for previous correlations; (ii) incorporate the strong condensing (densifying) effect of intercalated cholesterol, which is not addressed by previous models; and (iii) bring data sets quantifying the dramatically lower permeabilities of gel-phase bilayers into the fold of a unified framework with all the existing fluid-phase data. The key to achieving this level of universality is the introduction of a rigorously motivated minimum area per fatty acid chain substantially smaller than commonly considered.

The presentation is organized as follows. Two preliminary sections provide a succinct summary of the existing theoretical framework underpinning bilayer permeability, mostly emerging from the laboratory of Anderson, Xiang and coworkers, and motivate a revision of the normalized lipid density $\sigma$, a parameter arising in numerous past analyses. Thereafter we introduce the permeability data sets employed in our analysis, and collect necessary data from the lipid biophysics literature on the area per phospholipid chain. With these inputs, a systematic regression yields an explicit formula describing the dependence of $P^{\text {lip/w }}$ upon permeant size, as quantified by MW, and the new version of the normalized lipid density $\sigma$. The Discussion section comments on a number of aspects of the analysis.

\section{THEORETICAL FRAMEWORK}

Measured bilayer permeability coefficients $P^{\text {lip/w }}$ show enormous variations - over about 9 orders of magnitude - with variations in the permeating solute and in the phospholipid composition, temperature and cholesterol content of the bilayer. When plotted as a function of parameters like solute molecular weight (MW) or octanol/water partition coefficient $\left(K^{\text {oct } / \text { w }}\right)$, 
they give the appearance of baffling scatterings of points with almost no discernable trends. The key to extracting meaningful patterns (and usable correlations) from these data lies in: (i) considering a normalized permeability defined as the ratio of $P^{\text {lip/w }}$ to a suitable measure of solute lipophilicity within the barrier region of the bilayer; and (ii) identifying a parameter that appropriately quantifies the ratio of solute size to free (void) space between fatty acid chains, which is the key determinant of solute partitioning and mobility. Thus, our analysis is based on the equations

$$
\text { normalized permeability } F(\chi)=\frac{P^{\text {lip } / \mathrm{w}}}{\left.K^{\text {decadiene } / \mathrm{w}}\right|_{25^{\circ} \mathrm{C}} f_{\text {solvent }}}
$$

and

$$
\chi=\frac{(\mathrm{MW})^{n} \sigma}{1-\sigma} .
$$

A self-contained statement of the logic underlying them is provided in the Supporting Information for this article, and the reader is referred to Part $\mathrm{I}^{7}$ for further background.

Equation 1 reflects an important discovery made by Xiang, Anderson and coworkers based on correlations of permeability coefficients with various bulk solvent/water partition coefficients. These investigators identified 1,9-decadiene as a solvent which "precisely mimics the chemical selectivity of the barrier domain in egg lecithin bilayers," 11 and seems also to be a reasonably good mimic for DMPC-cholesterol bilayers, ${ }^{12,13}$ solidifying a conclusion emerging from previous reports. ${ }^{14-18}$ This result has been further substantiated ${ }^{19}$ and cited $^{9}$ more recently. In operational terms, the stated conclusion means that one should attempt to establish correlations for the ratio $P^{\text {lip/w }} /\left.K^{\text {decadiene } / w}\right|_{25^{\circ} \mathrm{C}}$ as opposed to $P^{\text {lip/w }}$ itself. ${ }^{7}$ In the absence of an experimental value, $\left.K^{\text {decadiene } / \mathrm{w}}\right|_{25^{\circ} \mathrm{C}}$ may be estimated for any solute using available 
empirical correlations. ${ }^{10,20}$ These correlations require Abraham solvation parameters ${ }^{21,22}$ as inputs, and can be expected to yield predictions of $\left.\log _{10} K^{\text {decadiene } / \mathrm{w}}\right|_{25^{\circ} \mathrm{C}}$ to within a root-meansquare error of $0.49 .^{20}$

The bulk chemical properties effectively modeled by 1,9-decadiene encompass both the partition coefficient for transfer of a solute from water to the bilayer interior, and its diffusion coefficient therein. For any particular solute, the symbol $f_{\text {solvent }}$ in Eq. 1 represents a correction factor accounting for variations of the bulk 1,9-decadiene/water partition with temperature $T$, and of the solute diffusivity in bulk 1,9-decadiene with $T$ and MW. Such a factor was first introduced by Xiang and Anderson ${ }^{13}$ who found that its inclusion improved the correlation of permeability data. Part $\mathrm{I}^{7}$ presents a detailed derivation of the formula

$$
f_{\text {solvent }}=\left(\frac{100}{\mathrm{MW}}\right)^{0.56} \times \exp \left[-5.5 \times 10^{3} \mathrm{~K}\left(\frac{1}{T}-\frac{1}{298.15 \mathrm{~K}}\right)\right]
$$

used there and in the present analysis. (This equation differs slightly from Eq. 23 of Part I in the inclusion of 100 as a reference molecular weight; the change is esthetic rather than substantive.)

The normalized permeability $F$, analogous to the "permeability decrement" $f$ introduced by Xiang, Anderson and coworkers, ${ }^{16,23-26}$ is strongly affected by the ordering of fatty acid chains near the head group region, which greatly restricts occupancy of and motion through the limited spaces between them by any given solute ("chain-ordering effects"). For a given solute, $F$ decreases with increasing lipid density. For a given lipid under given conditions (i.e., for a given lipid density), $F$ decreases with increasing solute size. Lipid density is quantified in terms of a relative ("normalized," "reduced") surface density defined by the equation

$$
\sigma=A_{0} / A, \quad A_{0}=40.8 \AA^{2},
$$


where $A$ denotes the area per phospholipid reckoned in the plane of the bilayer, and $A_{0}$ denotes the minimum area in the crystalline state ${ }^{16,23,27,28}$ ("hard-core minimum area"29). The value given for $A_{0}$ is the one usually stated, ${ }^{16,23,27,28}$ with slight variations. ${ }^{29}$

Equation 2 defines the correlating variable $\chi$ introduced in Part $\mathrm{I}^{7}$ that seems to be the most general and useful choice for purposes of quantifying variations in both solute size and lipid density within a common framework. The factor $(\mathrm{MW})^{n}$ is a measure of solute size. As discussed in the Supporting Information, MW raised to a power around $1 / 3$ tends to reflect the cross-sectional area that the solute takes up in the plane of the bilayer, whereas solute volume scales with MW raised to the power 0.94. The value of the exponent $n$ that provides the best correlation of existing permeability data is determined by our analysis (see Results below). The quantity $(1-\sigma) / \sigma$ has interpretations in terms of both free surface area and void volume in the bilayer. Thus, the ratio $\chi$ as given by Eq. 2 quantifies solute size vis-à-vis the average size of voids between fatty acid chains. The normalized permeability or chain-ordering factor $F$ is taken to be a function of the argument $\chi$ as a key determinant of solute partitioning into and mobility within the barrier region of the bilayer.

One of the key findings emerging from the extensive experimental work and enlightened analysis by Xiang, Anderson and coworkers cited in numerous places above and in the Supporting Information is that measured bilayer permeability coefficients tend to follow a universal relationship when cast in terms of a chain-ordering factor like $F$, and considered as a function of the lipid surface density $\sigma$, irrespectively of whether changes in $\sigma$ derive from variations in temperature or cholesterol concentration, ${ }^{13,16}$ or even phase (fluid or gel) of the bilayer. $^{16,23}$ Although successful correlations of chain ordering effects have been achieved for a 
number of permeability data sets, ${ }^{7,13,16,23}$ finding a truly universal formula describing all existing data (spanning numerous phosphatidylcholines, numerous solutes, wide variations in temperature and cholesterol content, and both fluid and gel phases) to high accuracy (i.e., with a low rootmean-square error in $\log _{10} P^{\text {lip/w}}$ ) has remained an elusive goal. We present such a formula for $F(\chi)$ below. As explained in the next section, the key to deriving it is a critical assessment and revision of the definition of the relative surface density $\sigma$.

\section{CRITIQUE OF $\sigma$ AS A CORRELATING VARIABLE}

The basic idea that lipid density as quantified by the relative surface density $\sigma$ is the key determinant of the chain-ordering factor (for a given solute) is sound. Furthermore, a value for $A_{0}$ around $40.8 \AA^{2}$ per phospholipid (or $\sim 20.4 \AA^{2}$ per fatty acid chain) is approximately correct. However, this value is not precise enough to deal with bilayers under conditions of high density, for reasons that emerge from a consideration of chain configurations at low temperatures.

\section{Gel-fluid transition}

Bilayers "melt" with increasing temperature from a more ordered (and therefore less accommodating and permeable) gel $\left(L_{\beta}\right)$ phase to a less ordered (and therefore more accommodating and permeable) fluid (liquid crystalline, $L_{\alpha}$ ) phase. A marked change in chain configuration occurs in traversing the gel-fluid transition (melting) temperature $T_{\mathrm{m}}$. Discussion could equally well be phrased in terms of "freezing" with decreasing temperature. Figure 1 characterizes this process in broad terms (cf. similar depictions ${ }^{30-32}$ ). 
Above $T_{\mathrm{m}}$ the two fatty acid chains of each phospholipid are in a state of considerable thermal motion and disorder, with an average alignment perpendicular to the plane of the bilayer. They take up considerably more area in the plane than the projected area of the head group to which they are attached. Thus, the head groups are more or less widely separated from each other. As the temperature is lowered, the spacing diminishes due to thermal contraction. This phenomenon is quantified by the coefficient of thermal area expansion $\alpha_{A}$, which is $\sim 3 \times 10^{-3}$ $\left({ }^{\circ} \mathrm{C}\right)^{-1}$ for most phospholipids. ${ }^{33,34}$ Around $T_{\mathrm{m}}$ the contraction approaches close packing of the head groups themselves. Here a problem arises. The chains would 'like' to get closer together as the temperature decreases further, owing to the van der Waals attractions between them, but their attachment to the head groups prevents them from doing so in an orientation perpendicular to the plane of the bilayer. Therefore the chains begin to tilt. This phenomenon allows a decrease in the inter-chain spacing while satisfying the constraint of covalent attachment to the head groups, which are now essentially at their minimum possible spacing. (This brief description glosses over the distinction between different gel phases existing below the main gel-fluid transition temperature $T_{\mathrm{m}}$, including rippled gel $\left(P_{\beta^{\prime}}\right)$, planar gel $\left(L_{\beta^{\prime}}\right)$ and subgel

phases, ${ }^{30,31,35-37}$ listed in order of decreasing temperature. As motivation for reflecting on the definition of $\sigma$, the main idea as stated above is sufficient, i.e., structural differences between these phases do not materially affect the analysis to follow.)

\section{Area per phospholipid and area per chain}

Gel-phase structure is quantified in detail for five saturated-chain phosphatidylcholines by Sun et al. ${ }^{38}$ (see especially their Table 1 and Figure 6). The actual inter-chain spacing is reflected in their data for the area per chain $A_{c}$. The degree of tilt is quantified by an angle $\theta$ measured 
relative to an axis normal to the plane of the bilayer. As temperature decreases, $A_{c}$ decreases and $\theta$ increases. These opposing effects balance each other almost perfectly to produce a very weak temperature dependence of the area per phospholipid $A$ (coefficient of thermal expansion $\alpha_{A}=2$ $\times 10^{-4}\left({ }^{\circ} \mathrm{C}\right)^{-1}$ and $4 \times 10^{-4}\left({ }^{\circ} \mathrm{C}\right)^{-1}$ for DPPC and DSPC, ${ }^{38}$ respectively, which is an order of magnitude lower than $\alpha_{A}$ for the fluid phase). It is important to emphasize that the area per chain $A_{\mathrm{c}}$ still varies significantly with temperature, somewhat more weakly than above $T_{\mathrm{m}}$ but at the same order of magnitude. For DPPC, for instance, $d A_{c} / d T=0.026 \AA^{2} /{ }^{\circ} \mathrm{C}$; given that $A_{c}=$ $20.2 \AA^{2}$ at $25^{\circ} \mathrm{C}$, this derivative is equivalent to a coefficient of thermal expansion for the area per chain $\left(A_{\mathrm{c}}\right)^{-1} d A_{\mathrm{c}} / d T=1.3 \times 10^{-3}\left({ }^{\circ} \mathrm{C}\right)^{-1}\left(\right.$ cf. $\alpha_{A} \sim 3 \times 10^{-3}\left({ }^{\circ} \mathrm{C}\right)^{-1}$ for the fluid phase). The key consequence of this phenomenon is that below $T_{\mathrm{m}}$ the area per phospholipid $A$ (or relative surface density $\sigma$ ) loses validity as a direct indicator of the actual spacing of fatty acid chains. This point is discussed further in the Supporting Information.

\section{Hard-core area per chain}

Sun et al.'s gel-phase data ${ }^{38}$ also bring to light a problem with use of the value $A_{0}=40.8 \AA^{2}$ in the definition of $\sigma$. In particular, at sufficiently low temperatures $A_{\mathrm{c}}$ dips below $(1 / 2) A_{0}=20.4$ $\AA^{2}$ (see their Table 1 and Figure 6, in which most of the determinations of $A_{c}$ are in fact below $20.4 \AA^{2}$ ). Thus, the true hard-core area per chain (denoted here by the symbol $A_{c, 0}$ ) must be somewhat smaller. It is important to calibrate this property properly, because it directly determines the amount of void space between fatty acid chains so crucial to chain ordering effects.

\section{Revised definition of $\sigma$}


We introduce a revised definition of the relative surface density $\sigma$ based on the area per chain $A_{c}$, and recalibrate its minimum hard-core value $A_{c, 0}$. In the fluid phase, $A_{c}$ is taken to be

$(1 / 2) A$, with data for $A$ drawn from Kučerka et al..$^{34}$ and other studies. ${ }^{33,39,40}$ (The factor of 2 arises because each phospholipid has two fatty acid chains. Although this is a valid formal assignment, the Supporting Information addresses an important subtlety related to it that warrants discussion, and is addressed there.) In the gel phase, data for $A_{\mathrm{c}}$ are drawn from Sun et al. ${ }^{38}$ cited above, as well as Tristram-Nagle et al. ${ }^{41}$ Also, instead of fixing $A_{\mathrm{c}, 0}$ at the value expected from precedent (i.e., $(1 / 2) A_{0}=20.4 \AA^{2}$ ), we rather take it to be an adjustable parameter determined by the criterion of best fitting bilayer permeability data. A robust value emerges by this procedure, as demonstrated in the Results and further remarked upon in the Discussion below. Thus, for any putative value of $A_{c, 0}$, the reduced surface density is defined by the twopart formula

$$
\begin{aligned}
& \sigma^{\prime}=\left\{\begin{array}{lll}
A_{\mathrm{c}, 0} /(A / 2), & T>T_{\mathrm{m}} & \text { (fluid phase), } \\
A_{\mathrm{c}, 0} / A_{\mathrm{c}}, & T<T_{\mathrm{m}} & \text { (gel phase), }
\end{array}\right. \\
& A_{\mathrm{c}, 0}=\text { adjustable parameter }<20.4 \AA^{2} .
\end{aligned}
$$

This revised definition of the relative surface density, distinguished with a prime $(/)$ superscript, supplants Eq. 4 in our analysis.

\section{METHODS}

Our overall method is to draw quantitative conclusions based on regression of $N=85$ permeability point data points reported by Xiang, Anderson and coworkers,, ,13-16,18,19,23,26 employing the revised definition of $\sigma$ (recast as $\sigma^{\prime}$ given by Eq. 5). 


\section{Permeability data}

Our analysis is based on a compilation of permeability data sets reported in a series of papers by Xiang, Anderson, and coworkers. It comprises measured values of $P^{\text {lip/w }}$ for: (1) 37 compounds in fluid-phase pure (cholesterol-free) egg lecithin or DOPC (regarded as equivalent) at 25 ${ }^{\circ} \mathrm{C} ; 9,14,15,18,19$ (2) acetic acid in fluid- and gel-phase DMPC and DPPC at various temperatures and cholesterol mole fractions; ${ }^{13,16}$ (3) several carboxylic acids (formic, acetic and butyric acids) in fluid- and gel-phase pure DPPC at various temperatures; ${ }^{23}$ and (4) silatecan 7-tbutyldimethylsilyl-10-hydroxycamptothecin (DB-67) lactone in gel-phase pure DSPC at $37^{\circ} \mathrm{C} .{ }^{26}$

Item (1) is exactly the same data set as that presented in Part I, ${ }^{7}$ Table 1 with one difference, namely the removal of two data points for $p$-methylhippuruc acid and $\alpha$-carbamoyl-pmethylhippuruc acid in egg phosphatidylcholine with 26 volume percent cholesterol. ${ }^{24}$ (Corresponding data for these two solutes in the pure phospholipid are retained, however). The points with cholesterol are not used here because of uncertainty about the effect of cholesterol on the partial specific area of phospholipid, a property needed for subsequent analysis. (In Part I we employed reported values of the apparent (as distinct from partial specific) area per phospholipid molecule ${ }^{42,43}$ for DOPC-cholesterol bilayers in our subsequent analysis.) This data set comes from papers by Xiang and Anderson, ${ }^{14,15}$ Mayer et al. ${ }^{18,19}$ and Cao et al. ${ }^{9}$ Because Part II, Table 1 states the values of $P^{\text {lip/w }}$ (together with other solute properties) and exhaustively catalogs all statements of each data point in the literature, it is unnecessary to repeat this table here. It serves the purpose of quantifying how permeability varies with changes in solute size in "relatively disordered bilayers, ${ }^{\prime 9}$ that is, a given low-density phospholipid (egg lecithin at $25^{\circ} \mathrm{C}$ ). 
The data embodied in items (2) and (3) are listed in detail in Table 1. The original sources actually list a significant number of data points not included in the table (and correspondingly our analysis). As in the previous paragraph, these exclusions do not imply any doubt about the permeability measurements. Rather, they reflect a lack of information on solute or bilayer properties needed for subsequent analysis. The specific exclusions are as follows. (i) All of Xiang and Anderson's data ${ }^{16}$ for DLPC and DSPC are omitted because they occur in mixtures with cholesterol, and we lack data on the partial specific areas of the phospholipid. (ii) All of Xiang and Anderson's data ${ }^{23}$ for propionic, valeric, isovaleric and trimethylacetic acids are excluded for lack of measured values of $K^{\text {decadiene } / \mathrm{w}} \mid{ }_{25^{\circ} \mathrm{C}}$ for these solutes. (iii) Data points reported by Xiang and Anderson ${ }^{13,16}$ for phospholipids with cholesterol are omitted if the temperature is below the melting temperature $T_{\mathrm{m}}$ of the pure phospholipid $\left(24^{\circ} \mathrm{C}\right.$ for DMPC, $41.4{ }^{\circ} \mathrm{C}$ for $\mathrm{DPPC}^{35,44}$ ). The reason is to be absolutely sure of avoiding coexistence between gel and liquid ordered $\left(L_{0}\right)$ phases in the phospholipid-cholesterol phase diagram. This point is addressed in detail in the Discussion. (iv) We also exclude data points for temperatures differing by less than $2{ }^{\circ} \mathrm{C}$ from the melting temperature $T_{\mathrm{m}}$ of the pure phospholipid. The reason is suggested by specific volume ${ }^{35,36}$ and permeability ${ }^{23}$ data, which show that gel-fluid phase transition is not perfectly sharp, but rather occurs over a small but finite temperature interval. We wish to be safely within the gel or fluid phase, and not within the sigmoidal transition zone. Only data points surviving these four filtering criteria are included in Table 1. Many of the excluded data points could be used if we were to introduce estimates of the properties needed for subsequent analysis $\left(\left.K^{\text {decadiene } / \mathrm{w}}\right|_{25^{\circ} \mathrm{C}}\right.$, partial specific areas of the phospholipid in phospholipidcholesterol mixtures). Our decision is not to corrupt our analysis with the additional errors arising from such estimates. Table 2 serves the purpose of quantifying how $P^{\text {lip/w }}$ varies with 
changes in lipid density (arising from changes in temperature, cholesterol mole fraction or phase) for a few given solutes (mostly acetic acid, with some data for formic and butyric acids).

The datum (4) is particularly valuable, because the combination of the second-largest molecular weight $(\mathrm{MW}=479)$ and the highest gel-phase lipid density conspire to produce by far the largest value of $\chi$ (see Eq. 2). Thus, the range of the data is extended to very strong chain ordering effects. This datum is included in Table 1, even though it is unrelated to data sets (2) and (3), because it must be listed somewhere. Thus, Table 1 here presents all data sets used in the present analysis beyond data set (1) (which is effectively presented in Part I, ${ }^{7}$ Table 1).

In their data reduction, Xiang, Anderson, and coworkers treat carefully the existence of unionized and ionized forms of their weak acid solutes, which leads to $\mathrm{pH}$ dependence of apparent permeability coefficients, as well as mass transfer resistances associated with unstirred layers in diffusion cells. Thus, all reported permeability coefficients cited here are values referring specifically to the unionized form of ionizable molecules, and to the intrinsic permeability of the bilayer itself.

The complete data set is presented in Figure 2 in the form of a $\log -\log$ plot of $P^{\text {lip/w }}$ as a function of $\left.K^{\text {decadiene } / \mathrm{w}}\right|_{25^{\circ} \mathrm{C}}$. The overall strong correlation between the ordinate and the abscissa is demonstrated by data set (1), rendered with open circles, for which variations in the chain-ordering factor arising from varying solute size produce only moderate scatter. It constitutes the basis for use of 1,9-decadiene as the model solvent noted above. Data sets (2) and (3), rendered with filled circles and squares, exhibit major deviations from the trend established by the low-density lipid (egg lecithin) data (dashed line) arising from variations in lipid density. This deviation becomes colossal for the datum (4), rendered as a filled diamond, which features 
the second-largest molecular weight on top of the highest (gel-phase) lipid density. Figure 2 here may be compared with Figure 1 of Part I. Here there is much more scatter (i.e., greater variation in chain-ordering effects) resulting from the inclusion of gel-phase results in data sets (2) and (3), as well as the extreme datum (4).

\section{Data on area per phospholipid}

For use with Eq. 5, Table 2 gives the expressions used to calculate the area per phospholipid $A$ in the fluid phase, and the area per chain $A_{c}$ in the gel phase, for pure phosphatidylcholines. These formulas are based on data reported in the lipid biophysics literature, ${ }^{33,34,38-41}$ identified in detail in the table.

For bilayers with cholesterol, $A$ is identified with the partial specific area of the phospholipid species as defined by Edholm and Nagle. ${ }^{45}$ Their model for DPPC bilayers with cholesterol has recently been corroborated quantitatively by a datum at $T=50{ }^{\circ} \mathrm{C}$ and $X_{\text {chol }}=$ 0.325 reported by Armstrong et al. ${ }^{46}$ using neutron diffraction in the plane of the bilayer. Values of the partial specific area of DPPC read from Edholm and Nagle's ${ }^{45}$ Figure $4\left(a_{\mathrm{DPPC}}\right.$ in their notation) are listed in Table 3 in the form of the ratio

$$
f_{\text {chol }} \equiv \frac{A(\operatorname{lip}, T, 0)}{A\left(\operatorname{lip}, T, X_{\text {chol }}\right)}
$$

in which $A(\operatorname{lip}, T, 0)$ denotes the area per phospholipid for the pure phospholipid. (An equivalent graph is presented by Armstrong et al. ${ }^{46}$ as their Figure 4). Table 3 also lists corresponding data for bilayers of DMPC with cholesterol at $30^{\circ} \mathrm{C}$ read from Pan et al.'s ${ }^{43}$ Figure 5. 


\section{Regression of data for the normalized permeability $F$}

Measured permeability coefficients are converted to the normalized permeability $F$ using Eqs. 1 and 3. For any given value of $A_{c, 0}$, the normalized surface density $\sigma^{\prime}$ is calculated from Eq. 5 . Thereafter, for any given value of the exponent $n$, the solute molecular weight MW and $\sigma^{\prime}$ combine to produce a numerical value of the correlating variable $\chi$ according to Eq. 2 . Molecular and lipid properties, and all derived quantities, are compiled in a Microsoft Excel spreadsheet $[\mathrm{O}(100$ rows by 100 columns $)]$, available from the authors upon request. The primary task is to determine the least-squares best fit of a straight line to the data in plots of $\log _{10} F$ as a function of $\chi$, because - as found in Part $\mathrm{I}^{7}$ and confirmed here $-F$ exhibits an exponential rather than power-law dependence upon $\chi$.

\section{Determination of the hard core area per chain $\boldsymbol{A}_{\mathrm{c}, 0}$ and the exponent $n$}

The preceding regression analysis was programmed in Fortran and carried out over a range evenly spaced values of $A_{c, 0}<20.4 \AA^{2}$, and evenly spaced values of $n$. The program picked out the pair of values $A_{\mathrm{c}, 0}$ and $n$ yielding the smallest root-mean-square error $(s)$ in the prediction of $\log _{10} F$, or the largest (squared) correlation coefficient $\left(r^{2}\right)$.

\section{RESULTS}

Both optimization criteria (minimizing $s$, maximizing $r^{2}$ ) yield the same pair of values of $A_{\mathrm{c}, 0}$ and $n$. The final correlation is given by the equation

$$
F=(14.4 \mathrm{~cm} / \mathrm{s}) \exp (-0.151 \chi)
$$




$$
\left(A_{\mathrm{c}, 0}=17.1 \AA^{2}, n=0.51 ; N=85, s=0.3024, r^{2}=0.9332\right) .
$$

Although the indicated values of $A_{c, 0}$ and $n$ are the preferred ones, nearby values also yield very reasonable fits of the data.

Figures 3 and 4 are graphical presentations of the final correlation in two forms, namely a semi-log plot of $F$ as a function of $\chi$, and a log-log plot of calculated versus experimental permeability coefficients, respectively. Vis-à-vis Figure 2, Figure 5 demonstrates how division by the chain ordering factor $F(\chi)$ goes very far toward collapsing all the permeability data much more closely onto a universal dependence upon $\left.K^{\text {decadiene } / \mathrm{w}}\right|_{25^{\circ} \mathrm{C}}$ (As quantified in the Supporting Information, $f_{\text {solvent }}$ plays a useful but minor role in this collapse.)

\section{DISCUSSION}

The preceding analysis has established a basis for successful correlation of an extensive 85-point data set on phospholipid bilayer permeability that has accreted in the seminal work of Xiang, Anderson and coworkers cited repeatedly above. We recap all the equations needed to predict the permeability coefficient $P^{\text {lip/w }}$ in Table 4 for convenience. "Successful" means that $\log _{10} P^{\text {lip/w }}$ is predicted with a root-mean-square error of 0.30 if $K_{\text {decadiene/w }}$ is known; loosely speaking one can say that the value of $P^{\text {lip/w }}$, which varies over 9 orders of magnitude in the data set considered, is predictable to within a factor of about 2 on average.

The key to unifying all the data, in particular including 17 newly considered points for high-density gel-phase bilayers, is a suitable redefinition of the relative surface density $\sigma^{\prime}$ using a value of the hard-core area per chain $A_{c, 0}=17.1 \AA^{2}$ significantly smaller than one-half of the 
accepted value $A_{0}=40.8 \AA^{2}$ for the minimum area per phospholipid in the crystalline state. Relative to Part I, ${ }^{7}$ there is a further addition to the data set considered here, namely 11 points for fluid-phase bilayers of DPPC without and with cholesterol.

The equations in Table 4 are robust in the sense that they unite data measured for five phospholipids (egg lecithin, DOPC, DMPC, DPPC and DSPC), cholesterol mole fractions ranging from 0 to 0.4 , and both fluid and gel phases. With the framework established here, measurements made for these diverse sets of conditions all tell a consistent story.

\section{Role of present analysis}

Previous correlations for $P^{\text {lip/w }}$ apply to low-density fluid-phase phospholipids like egg lecithin and DOPC at room temperature without cholesterol ${ }^{9,10}$ (see open circles in Figure 2). For such lipids $P^{\text {lip/w }}$ and $\left.K^{\text {decadiene } / w}\right|_{25^{\circ} \mathrm{C}}$ are almost synonymous, and the tasks of predicting these two coefficients are essentially equivalent. In this context it is significant that investigators tend to develop correlations for both coefficients in parallel. ${ }^{9,10}$ Aside from experimental uncertainty, a small amount of scatter exists due to variations in MW (which cause correspondingly small variations in the chain ordering factor at low density). However, the main source of variations in $P^{\mathrm{lip} / \mathrm{w}}$, over some 9 orders of magnitude among different solutes, is variations in the pertinent measure of lipophilicity, namely $\left.K^{\text {decadiene } / \mathrm{w}}\right|_{25^{\circ} \mathrm{C}}$. All told, the objective of obtaining reasonable predictions of $P^{\text {lip/w }}$ in low-density phospholipid bilayers can be regarded as a largely solved problem, subject to evolutionary improvement.

The outstanding challenge has been to account in a systematic way for variations in $P^{\mathrm{lip} / \mathrm{w}}$ that arise from variations in temperature, cholesterol mole fraction, or phase (fluid or gel) 
of the bilayer. This challenge is answered by the present analysis. Incorporation of large amounts of cholesterol, or transition to the gel phase, can greatly increase the lipid density (see values of $\sigma^{\prime}$ in Table 1), with concomitant large decreases in the chain ordering factor. As shown by Figure 2, chain ordering effects can cause variations in $P^{\text {lip/w }}$ from approximately +1 to -8 on a $\log _{10}$ scale relative to the baseline established by the low-density data. Thus, chain ordering can lead to variations as large as those deriving from lipophilicity once gel-phase data are included in the analysis.

For solutes for which no experimental measurement of $\left.K^{\text {decadiene } / \mathrm{w}}\right|_{25^{\circ} \mathrm{C}}$ is available, the errors in estimating $\left.K^{\text {decadiene } / \mathrm{w}}\right|_{25^{\circ} \mathrm{C}}$ and the chain ordering factor $F$ would be compounded in calculating $P^{\text {lip/w }}$ according to Eq. 1 . This point has been discussed previously (see Part I, ${ }^{7}$ Table 6, Figure 6 and associated text).

\section{Hard-core area per chain $\boldsymbol{A}_{\mathrm{c}, 0}$}

The optimal value $A_{\mathrm{c}, 0}=17.1 \AA^{2}$ represents the minimum area per chain that best correlates the extensive permeability data set considered. This effective value vis-à-vis the concept of $A_{\mathrm{c}, 0}$ as a structural parameter that is measurable in principle is discussed in the Supporting Information.

\section{Solute molecular weight-dependence of permeability}

The final exponent $n=0.51$ in the definition of $\chi($ Eq. 2$)$ is closer to the value $\sim 0.33$ associated with free surface area theory than to the value 0.94 associated with free volume theory. Consideration of a broader data set reverses the opposite conclusion reached in Part I on this point. 


\section{Phase behavior}

The Supporting Information summarizes aspects of phospholipid-cholesterol phase behavior pertinent to application of the present analysis to various subsets of the $\left(T, X_{\text {chol }}\right)$ parameter space. Figure 6 schematizes the most important ideas with reference to the phase diagram for DPPC-cholesterol bilayers, ${ }^{47}$ with a view toward providing practical guidance for the estimation of $P^{\text {lip/w }}$. Its most important features are the regions of two-phase coexistence between fluid $\left(L_{\alpha}\right)$ and liquid ordered $\left(L_{\mathrm{o}}\right)$ phases above $T_{\mathrm{m}}$, and between gel $\left(L_{\beta}\right)$ and liquid ordered $\left(L_{\mathrm{o}}\right)$ phases below $T_{\mathrm{m}}$. The small $L_{\alpha}-L_{\beta}$ two-phase region at temperatures near $T_{\mathrm{m}}$ and low cholesterol mole fractions $\left(X_{\text {chol }} \leq \sim 0.07\right.$ ) would rarely be encountered in natural and experimental settings given its diminutive size. The difference between $T_{\mathrm{m}}$ and the temperature of three-phase $L_{\alpha}-L_{\beta}-L_{0}$ coexistence is small, and we regard them as essentially synonymous in broadly dividing the temperature axis into fluid and gel subsets. Indeed, the $L_{\alpha}-L_{\beta}$ two-phase region is sometimes rendered simply as a small horizontal line segment (see Xiang and Anderson's ${ }^{48}$ Figure 5).

The red boxes indicate regions in which (1) the model definitely applies because permeability measurements in these regions entered the analysis (with the exclusion of a few available measurements very near $T_{\mathrm{m}}$ as discussed above). As justified in the Supporting Information, they include the region of coexistence between fluid $\left(L_{\alpha}\right)$ and liquid ordered $\left(L_{\mathrm{o}}\right)$ phases above $T_{\mathrm{m}}$.

Green boxes indicate regions in which (2) the model could be applied in principle if detailed data on the cholesterol-dependence of the area per chain were available for gel $\left(L_{\beta}\right)$ and liquid ordered $\left(L_{\mathrm{o}}\right)$ phases, on a par with the papers cited above for fluid-phase phospholipid- 
cholesterol mixtures. ${ }^{43,45,46}$ These regions lie below $T_{\mathrm{m}}$ and outside the $L_{\beta}-L_{\mathrm{o}}$ two-phase region. Applicability of the model is somewhat speculative because no data from these regions entered the fitting process. (Although some permeability data are available, ${ }^{48}$ requisite area-per-chain data to support the analysis are lacking.) Nevertheless, it is supported by the fact that the model is valid over wide ranges of temperature and cholesterol mole fraction, and for both fluid and gel phases. The single-phase gel $\left(L_{\beta}\right)$ region to the left of the phase boundary is quite narrow ( $X_{\text {chol }}$ $\leq \sim 0.07$ ), and one could be forgiven for approximating the mixture permeability in this region as that of the pure phospholipid. In particular, plots of $\log _{10} P^{\text {lip/w }}$ as a function of $X_{\text {chol }}$ are horizontal for acetic acid, and have smallish slopes for trimethylacetic acid, in the data reported by Xiang and Anderson ${ }^{48}$ for this region (see their Figures 4 and 6). Possible use of this approximation becomes a moot point below the transition temperature between rippled $\left(P_{\beta^{\prime}}\right)$ and planar $\left(L_{\beta^{\prime}}\right)$ gel phases, because $X_{\text {chol }}$ for the $L_{\beta^{\prime}}$ phase in equilibrium with the $L_{\mathrm{o}}$ phase is essentially indistinguishable from zero (see Xiang and Anderson's ${ }^{48}$ Figure 5). The permeability of the liquid ordered $\left(L_{\mathrm{o}}\right)$ phase decreases as a function of $X_{\mathrm{chol}}$, starting from a value at the right phase boundary (at $X_{\mathrm{chol}} \sim 0.22$ ) that is typically about an order of magnitude greater than the permeability of the gel $\left(L_{\beta}\right)$ phase.

The blue box indicates the region in which (3) permeability coefficients should be calculated using the lever rule $e^{47-50}$ together with the permeability coefficients of the gel and liquid ordered phases at the phase boundaries (at $X_{\text {chol }} \sim 0.07$ and 0.22 , respectively). Our analysis could supply such a calculation with the requisite estimates of $P^{\text {lip/w }}$ for these two coexisting phases. (The small $L_{\alpha}-L_{\beta}$ two-phase region would also be a region of this type.) 
Rational estimates of permeability coefficients require knowledge of the $\left(T, X_{\text {chol }}\right)$ phase diagram for the phospholipid-cholesterol mixture of interest, which may or may not be available. The discussion above and in the Supporting Information is phrased in terms of binary mixtures (i.e., one phospholipid plus cholesterol). A number of investigations have also elucidated phase diagrams for binary mixtures of two phospholipids ${ }^{48}$ and ternary mixtures of two phospholipids plus cholesterol. ${ }^{51-53}$ We speculate that the present model might yield reasonable estimates of $P^{\mathrm{lip} / \mathrm{w}}$ whenever the lipid mixture exists as a single phase, with the caveat that it requires specific knowledge of the lipid density. Based on the published binary and ternary phase diagrams cited, it seems that phospholipid-cholesterol mixtures having the high cholesterol mole fractions typical of cell plasma membranes (30-50 mole percent ${ }^{54-58}$ ) might often exist as a single phase.

\section{Comparison of the present correlation with that developed in Part I}

It is worthwhile to compare the correlation developed here, summarized in Table 4, with that developed in Part I. ${ }^{7}$ As shown in the Supporting Information, the present correlation is roughly equivalent to the previous one for fluid-phase bilayers, for which the values of $\chi$ corresponding to available permeability data points lie within interval $4<\chi<24$ based on the revised definitions of $\sigma$ (recast as $\sigma^{\prime}$ given by Eq. 5) and $\chi$ (given by Eq. 2 with $n=0.51$ ). This statement applies to both the representation of the dependence of normalized permeability $F$ upon area per phospholipid $A$, and the root-mean-square error in predicting $\log _{10} P^{\operatorname{lip} / w}$. However, the present correlation greatly extends the range of applicability to the gel phase $(35<$ $\chi<131)$, whereas the previous one cannot be applied to this phase at all.

\section{Inputs from the lipid biophysics literature}


In principle, the effect of cholesterol on phospholipid area, as quantified by $f_{\text {chol }}$ defined by Eq. 6, depends on temperature. At present, the scientific community is lucky to have available the data summarized in Table 3 , which is limited to a single temperature $\left(30^{\circ} \mathrm{C}\right.$ for DMPC, ${ }^{43} 50{ }^{\circ} \mathrm{C}$ for $\mathrm{DPPC}^{45,46}$ ). In analyzing permeability data for bilayers with cholesterol, we first calculate the pure-phospholipid area $A$ using the equations in Table 2, and then divide the value obtained by the appropriate value of $f_{\text {chol }}$ listed in Table 3 . This procedure amounts to assuming that $f_{\text {chol }}$ does not vary with temperature. Some error estimates related to this procedure, for which there is presently no alternative, are offered in Part I (see p. 2028 and Supporting Information). The fact of having to make this approximation underscores the importance of further studies to quantify the dependence of $A$ upon $X_{\text {chol }}$ at various temperatures.

\section{CONCLUSIONS}

The main conclusions of our analysis are as follows. First, existing data on phospholipid and phospholipid-cholesterol bilayer permeability, supported by density determinations from the lipid biophysics literature, establish a basis for predicting variations in $\log _{10} P^{\text {lip/w }}$ over about nine orders of magnitude arising from variations in solute size and lipid density to within a rootmean-square error of 0.30 (or variations in $P^{\text {lip/w }}$ to within a factor of about 2). Second, fluidand gel-phase data are fully consistent with each other, and can be described by a single exponential relationship, if the hard-core area per chain $A_{\mathrm{c}, 0}$, considered as an effective value, is taken to be $17.1 \AA^{2}$. This result is significantly smaller than one-half of the accepted value $A_{0}=$ $40.8 \AA^{2}$ for the minimum area per phospholipid in the crystalline state. All equations needed to predict $P^{\text {lip/w }}$ are listed in Tables $2-4$. 


\section{ACKNOWLEDGMENTS}

The authors gratefully acknowledge support of this work from the US National Science Foundation (NSF) GOALI program. The conclusions drawn here reflect the opinions of the authors and have not been endorsed by NSF. 


\section{REFERENCES CITED}

1. Balani SK, Miwa GT, Gan LS, Wu JT, Lee FW 2005. Strategy of utilizing in vitro and in vivo ADME tools for lead optimization and drug candidate selection. Curr Top Med Chem 5(11):1033-1038.

2. Mandagere AK, Thompson TN, Hwang K-K 2002. Graphical model for estimating oral bioavailability of drugs in humans and other species from their Caco-2 permeability and in vitro liver enzyme metabolic stability rates. J Med Chem 45(2):304-311.

3. Kerns EH, Di L, Petusky S, Farris M, Ley R, Jupp P 2004. Combined application of parallel artificial membrane permeability assay and Caco-2 permeability assays in drug discovery. J Pharm Sci 93(6):1440-1453.

4. Poet TS, McDougal JN 2002. Skin absorption and human risk assessment. Chem-Biol Interact 140(1):19-34.

5. Fitzpatrick D, Corish J, Hayes B 2004. Modelling skin permeability in risk assessmentthe future. Chemosphere 55(10):1309-1314.

6. Centers for Disease Control and Prevention. Fourth National Report on Human Exposure to Environmental Chemicals, 2009. Accessed August 29, 2015, at: http://www.cdc.gov/exposurereport.

7. Nitsche JM, Kasting GB 2013. Permeability of fluid-phase phospholipid bilayers: assessment and useful correlations for permeability screening and other applications. J Pharm Sci 102(6):2005-2032.

8. Fujikawa M, Ano R, Nakao K, Shimizu R, Akamatsu M 2005. Relationships between structure and high-throughput screening permeability of diverse drugs with artificial membranes: Application to prediction of Caco-2 cell permeability. Bioorg Med Chem 13(15):4721-4732.

9. Cao Y, Xiang T-X, Anderson BD 2008. Development of structure-lipid bilayer permeability relationships for peptide-like small organic molecules. Mol Pharm 5(3):371-388.

10. Abraham MH, Acree WE, Jr. 2012. Linear free-energy relationships for water/hexadec-1ene and water/deca-1,9-diene partitions, and for permeation through lipid bilayers; comparison of permeation systems. New J Chem 36(9):1798-1806.

11. Mayer PT, Anderson BD 2002. Transport across 1,9-decadiene precisely mimics the chemical selectivity of the barrier domain in egg lecithin bilayers. J Pharm Sci 91(3):640-646.

12. Xiang T-X, Anderson BD 1995. Development of a combined NMR paramgnetic ioninduced line-broadening/dynamic light scattering method for permeability measurements across lipid bilayer membranes. J Pharm Sci 84(11):1308-1315.

13. Xiang T-X, Anderson BD 1995. Phospholipid surface density determines the partitioning and permeability of acetic acid in DMPC:cholesterol bilayers. J Membr Biol 148(2):157-167.

14. Xiang T-X, Anderson BD 1994. Substituent contributions to the transport of substituted p-toluic acids across lipid bilayer membranes. J Pharm Sci 83(10):1511-1518.

15. Xiang T-X, Anderson BD 1994. The relationship between permeant size and permeability in lipid bilayer membranes. J Membr Biol 140(2):111-122.

16. Xiang T-X, Anderson BD 1997. Permeability of acetic acid across gel and liquidcrystalline lipid bilayers conforms to free-surface-area theory. Biophys J 72(1):223-237.

17. Xiang T-X, Xu Y-H, Anderson BD 1998. The barrier domain for solute permeation varies with lipid bilayer phase structure. J Membr Biol 165(1):77-90. 
18. Mayer PT, Xiang T-X, Anderson BD 2000. Independence of substituent contributions to the transport of small-molecule permeants in lipid bilayer. AAPS Pharmsci 2(2):art. no. 14.

19. Mayer PT, Xiang T-X, Niemi R, Anderson BD 2003. A hydrophobicity scale for the lipid bilayer barrier domain from peptide permeabilities: nonadditivities in residue contributions. Biochemistry 42(6):1624-1636.

20. Nitsche JM, Kasting GB 2013. A correlation for 1,9-decadiene/water partition coefficients. J Pharm Sci 102(1):136-144.

21. Abraham MH 1993. Scales of solute hydrogen-bonding: their construction and aplication to physicochemical and biochemical processes. Chem Soc Rev 22(2):73-83.

22. Abraham MH, Ibrahim A, Zissimos AM, Zhao YH, Comer J, Reynolds DP 2002. Application of hydrogen bonding calculations in property based drug design. Drug Discov Today 7(20):1056-1063.

23. Xiang T-X, Anderson BD 1998. Influence of chain ordering on the selectivity of dipalmitoylphosphatidylcholine bilayer membranes for permeant size and shape. Biophys $\mathbf{J}$ 75(6):2658-2671.

24. Xiang T-X, Chen J, Anderson BD 2000. A quantitative model for the dependence of solute permeability on peptide and cholesterol content in biomembranes. J Membr Biol 177(2):137-148.

25. Xiang T-X, Anderson BD 2006. Liposomal drug transport: A molecular perspective from molecular dynamics simulations in lipid bilayers. Adv Drug Deliv Rev 58(12-13):1357-1378.

26. Joguparthi V, Xiang T-X, Anderson BD 2008. Liposome transport of hydrophobic drugs: Gel phase lipid bilayer permeability and partitioning of the lactone form of a hydrophobic camptothecin, DB-67. J Pharm Sci 97(1):400-420.

27. De Young LR, Dill KA 1988. Solute partitioning into lipid bilayer membranes. Biochemistry 27(14):5281-5289.

28. De Young LR, Dill KA 1990. Partitioning of nonpolar solutes into bilayers and amorphous $n$-alkanes. J Phys Chem 94(2):801-809.

29. Cantor RS, Dill KA 1986. Theory for the equation of state of phospholipid monolayers. Langmuir 2(3):331-337.

30. Evans E, Needham D 1987. Physical properties of surfactant bilayer membranes: thermal transitions, elasticity, rigidity, cohesion, and colloidal interactions. J Phys Chem 91(16):42194228.

31. Needham D, Evans E 1988. Structural and mechanical properties of giant lipid (DMPC) vesicle bilayers from $20^{\circ} \mathrm{C}$ below to $10{ }^{\circ} \mathrm{C}$ above the liquid crystal-crystalline phase transition at $24^{\circ} \mathrm{C}$. Biochemistry 27(21):8261-8269.

32. Sun W-J, Suter RM, Knewtson MA, Worthington CR, Tristram-Nagle S, Zhang R, Nagle JF 1994. Order and disorder in fully hydrated unoriented bilayers of gel phase dipalmitoylphosphatidylcholine. Phys Rev E 49(5):4665-4676.

33. Nagle JF, Tristram-Nagle S 2000. Structure of lipid bilayers. Biochim Biophys Acta 1469(3):159-195.

34. Kučerka N, Nieh M-P, Katsaras J 2011. Fluid phase lipid areas and bilayer thicknesses of commonly used phosphatidylcholines as a function of temperature. Biochim Biophys Acta 1808(11):2761-2771.

35. Nagle JF, Wilkinson DA 1978. Lecithin bilayers. Density measurements and molecular interactions. Biophys J 23(2):159-175. 
36. Tristram-Nagle S, Nagle JF 2004. Lipid bilayers: thermodynamics, structure, fluctuations, and interactions. Chem Phys Lipids 127(1):3-14.

37. Eeman M, Deleu M 2010. From biological membranes to biomimetic model membranes. Biotechnol Agron Soc Environ 14(4):719-736.

38. Sun W-J, Tristram-Nagle S, Suter RM, Nagle JF 1996. Structure of gel phase saturated lecithin bilayers: temperature and chain length dependence. Biophys J 71(2):885-891.

39. Petrache HI, Tristram-Nagle S, Nagle JF 1998. Fluid phase structure of EPC and DMPC bilayers. Chem Phys Lipids 95(1):83-94.

40. Kučerka N, Nagle JF, Sachs JN, Feller SE, Pencer J, Jackson A, Katsaras J 2008. Lipid bilayer structure determined by the simultaneous analysis of neutron and X-ray scattering data. Biophys J 95(5):2356-2367.

41. Tristram-Nagle S, Liu Y, Legleiter J, Nagle JF 2002. Structure of gel phase DMPC determined by X-ray diffraction. Biophys J 83(6):3324-3335.

42. Mathai JC, Tristram-Nagle S, Nagle JF, Zeidel ML 2008. Structural determinants of water permeability through the lipid membrane. J Gen Physiol 131(1):69-76.

43. Pan J, Tristram-Nagle S, Nagle JF 2009. Effect of cholesterol on structural and mechanical properties of membranes depends on lipid chain saturation. Phys Rev E 80(2):021931.

44. Greenwood AI, Tristram-Nagle S, Nagle JF 2006. Partial molecular volumes of lipids and cholesterol. Chem Phys Lipids 143(1-2):1-10.

45. Edholm O, Nagle JF 2005. Areas of molecules in membranes consisting of mixtures. Biophys J 89(3):1827-1832.

46. Armstrong CL, Marquardt D, Dies H, Kučerka N, Yamani Z, Harroun TA, Katsaras J, Shi A-C, Rheinstädter MC 2013. The observation of highly ordered domains in membranes with cholesterol. PLoS One 8(6):e66162.

47. Vist MR, Davis JH 1990. Phase equilibria of cholesterol/dipalmitoylphosphatidylcholine mixtures: ${ }^{2} \mathrm{H}$ nuclear magnetic resonance and differential scanning calorimetry. Biochemistry 29(2):451-464.

48. Xiang T-X, Anderson BD 1998. Phase structures of binary lipid bilayers as revealed by permeability of small molecules. Biochim Biophys Acta 1370(1):64-76.

49. Chiang Y-W, Costa-Filho AJ, Freed JH 2007. Dynamic molecular structure and phase diagram of DPPC-Cholesterol binary mixtures: a 2D-ELDOR study. J Phys Chem B 111(38):11260-11270.

50. Heerklotz H, Tsamaloukas A 2006. Gradual change or phase transition: Characterizing fluid lipid-cholesterol membranes on the basis of thermal volume changes. Biophys J 91(2):600607.

51. Veatch SL, Keller SL 2003. Separation of liquid phases in giant vesicles of ternary mixtures of phospholipids and cholesterol. Biophys J 85(5):3074-3083.

52. Veatch SL, Keller SL 2005. Seeing spots: Complex phase behavior in simple membranes. Biochim Biophys Acta 1746(3):172-185.

53. Veatch SL 2007. From small fluctuations to large-scale phase separation: Lateral organization in model membranes containing cholesterol. Sem Cell Develop Biol 18(5):573582.

54. Gallová J, Uhríková D, Islamov A, Kuklin A, Balgavý P 2004. Effect of cholesterol on the bilayer thickness in unilamellar extruded DLPC and DOPC liposomes: SANS contrast variation study. Gen Physiol Biophys 23(1):113-128. 
55. Huang T-H, Lee CWB, Das Gupta SK, Blume A, Griffin RG 1993. A ${ }^{13} \mathrm{C}$ and ${ }^{2} \mathrm{H}$ nuclear magnetic resonance study of phosphatidylcholine/cholesterol interactions: Characterization of liquid-gel phases. Biochemistry 32(48):13277-13287.

56. Pan J, Mills TT, Tristram-Nagle S, Nagle JF 2008. Cholesterol perturbs lipid bilayers nonuniversally. Phys Rev Lett 100(19):198103.

57. Pandit SA, Bostick D, Berkowitz ML 2004. Complexation of phosphatidylcholine lipids with cholesterol. Biophys J 86(3):1345-1356.

58. Cournia Z, Ullmann GM, Smith JC 2007. Differential effects of cholesterol, ergosterol and lanosterol on a dipalmitoyl phosphatidylcholine membrane: a molecular dynamics simulation Study. J Phys Chem B 111(7):1786-1801. 


\section{FIGURE CAPTIONS}

Figure 1. Simplified generic representation of the arrangement of fatty acid chains at temperatures above, at and below the gel-fluid transition ("melting") temperature $T_{\mathrm{m}}$, similar to Evans and Needham's ${ }^{30}$ Figure 3, Needham and Evans's ${ }^{31}$ Figure 1, and Sun et al.'s ${ }^{32}$ Figure 2. Here the ripple phase ${ }^{30,31}$ is not explicitly represented.

Figure 2. Log-log plot showing the correlation of $P^{\text {lip } / \mathrm{w}}$ with $\left.K^{\text {decadiene } / \mathrm{w}}\right|_{25^{\circ} \mathrm{C}}$. Open circles represent 37 solutes in low-density phospholipids (egg lecithin and DOPC; see Part I, ${ }^{7}$ Table 1). The dashed line represents the trend established by these data. Filled symbols represent the data in Table 2, which covers temperature-, cholesterol- and phase (fluid or gel)-dependent variations in phospholipid surface density for three solutes (formic, acetic and butyric acids) in DMPC and DPPC, plus a single datum for the solute DB-67 lactone in gel-phase pure DSPC. Among filled symbols, squares and circles represent measurements for DMPC and DPPC, respectively. Small and large symbols distinguish fluid and gel phases, respectively. Listed in order of increasing $\left.K^{\text {decadiene } / \mathrm{w}}\right|_{25^{\circ} \mathrm{C}}$, data for the solutes formic acid, acetic acid and butyric acid can be distinguished from each other because they occur as vertical columns of points. In the electronic version of this article these solutes are also distinguished by use of grey, black and green colors, respectively. The single datum for DB-67 lactone in gel-phase pure DSPC is rendered as a (blue) diamond.

Figure 3. Dependence of the chain ordering factor $F$, calculated from experimental permeability data according to Eq. 1, upon the correlating variable $\chi$ defined by Eq. 2 . Significance of markers used for points is as in Figure 2. Calculations are carried out with the 
final optimized parameters $A_{\mathrm{c}, 0}=17.1 \AA^{2}$ in Eq. 5 and $n=0.51$ in Eq. 2. The solid line represents Eq. 7.

Figure 4. Quality of fit provided by the equations in Table 4. Significance of markers used for points is as in Figure 2. The dotted line is the line of perfect fit.

Figure 5. Log-log plot showing the correlation of $P^{\text {lip/w }}$ with $\left.K^{\text {decadiene } / \mathrm{w}}\right|_{25^{\circ} \mathrm{C}}$ if the former quantity is normalized with factors accounting for temperature and solute MW dependencies of bulk partition and diffusion coefficients in 1,9-decadiene $\left(f_{\text {solvent }}\right)$, and solute MW and phospholipid surface density dependencies of the chain ordering factor $(F)$. Significance of markers used for points, as well as dashed line, is as in Figure 2.

Figure 6. Semiquantitative phase diagram for DPPC-cholsterol bilayers. Red boxes (labeled with the numeral " 1 " as in the text) indicate regions in which the present model definitely applies because permeability measurements in these regions entered the analysis. Green boxes ("2") indicate regions in which the model could be applied in principle if detailed data on the cholesterol-dependence of the area per chain were available. The blue box ("3") indicates the region in which permeability coefficients should be calculated using the lever rule together with the permeability coefficients of the gel and liquid ordered phases at the phase boundaries (at $X_{\text {chol }} \sim 0.07$ and 0.22 , respectively). The present model could supply such a calculation with the requisite estimates of $P^{\mathrm{lip} / \mathrm{w}}$ for these two coexisting phases. 


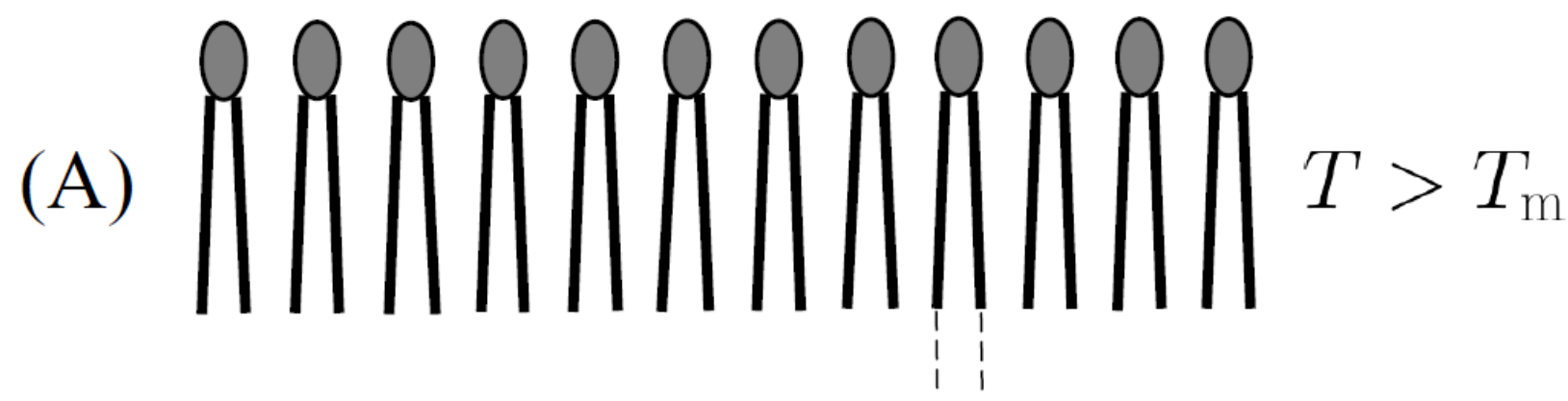
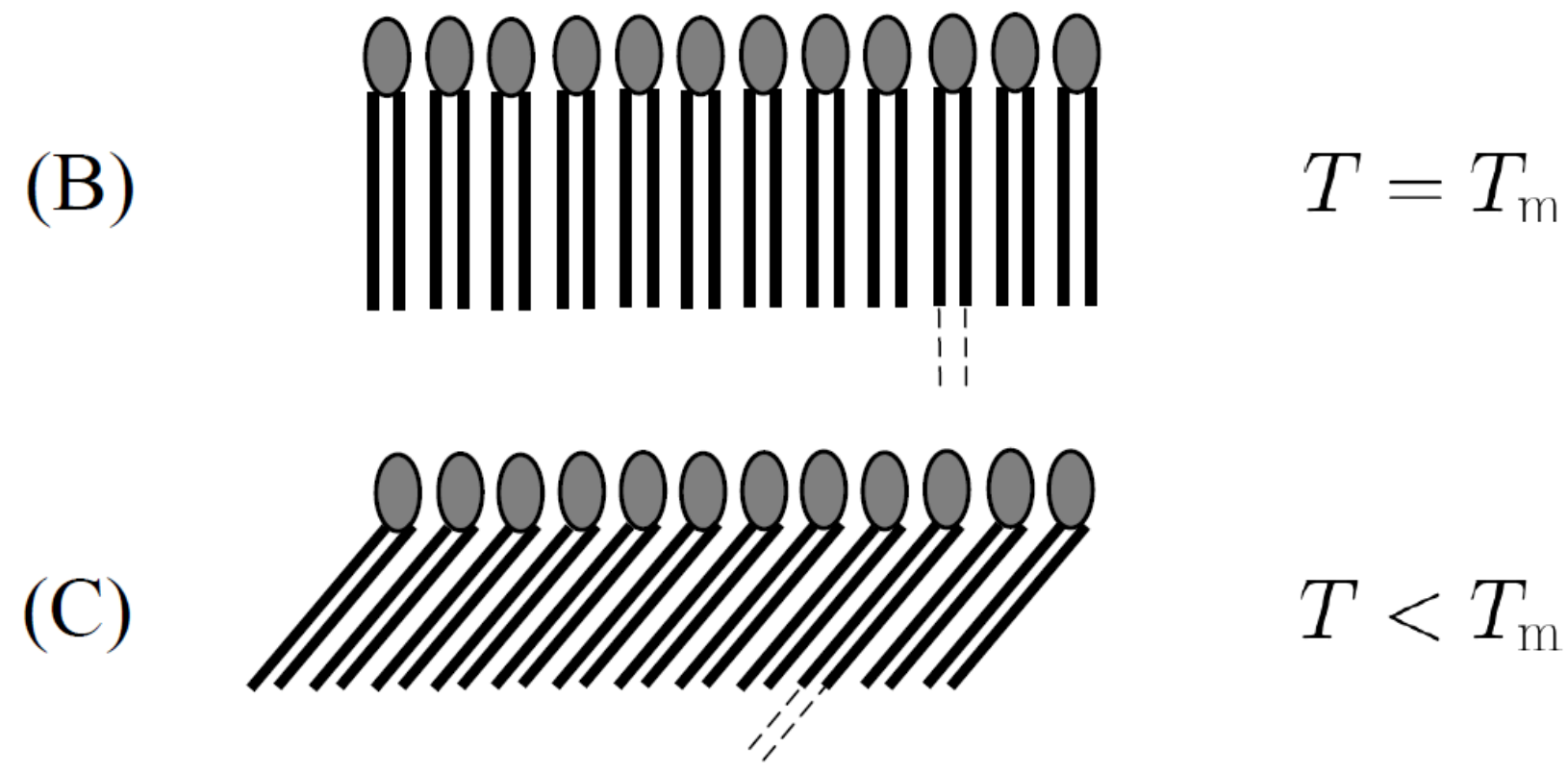


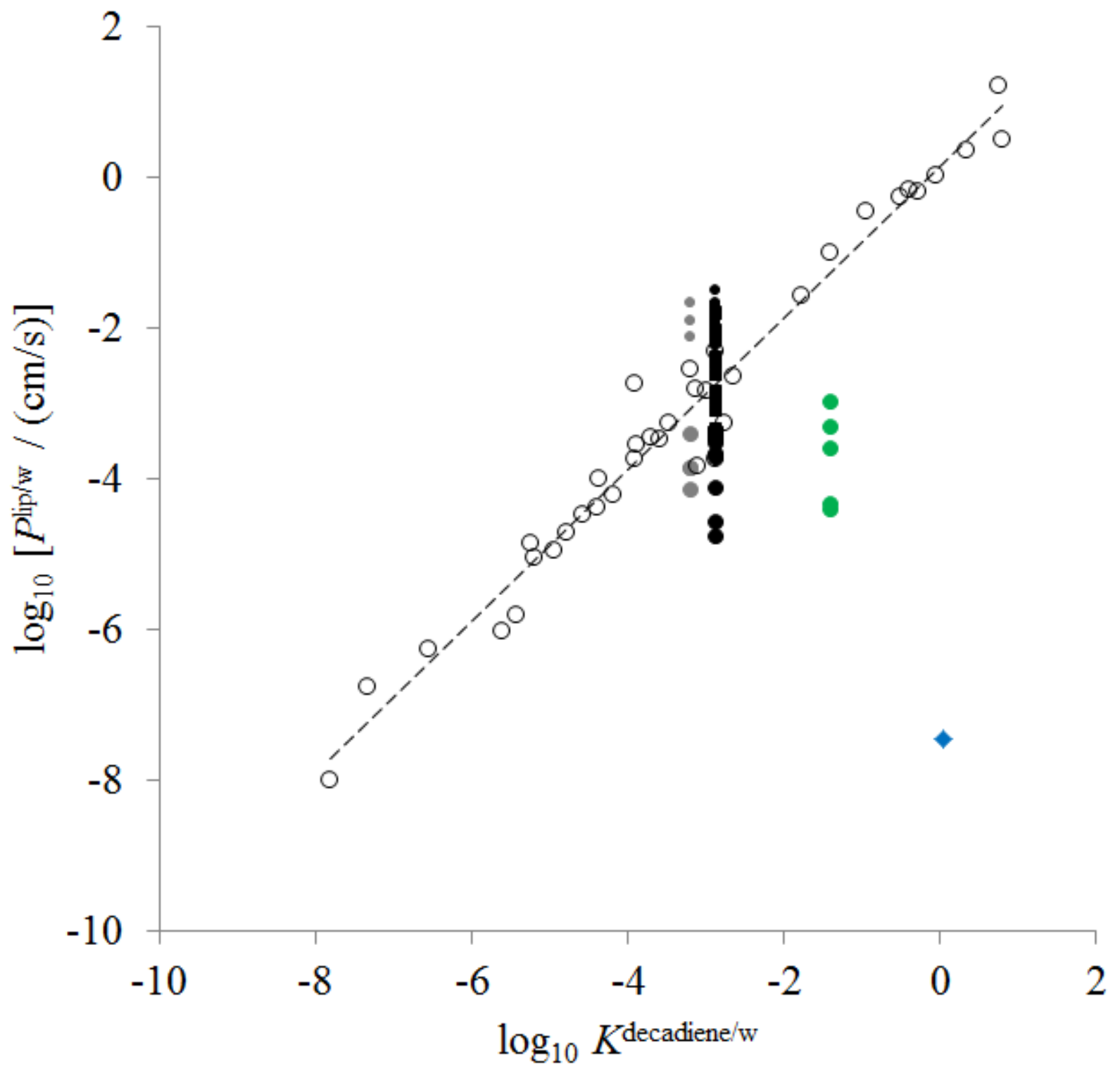




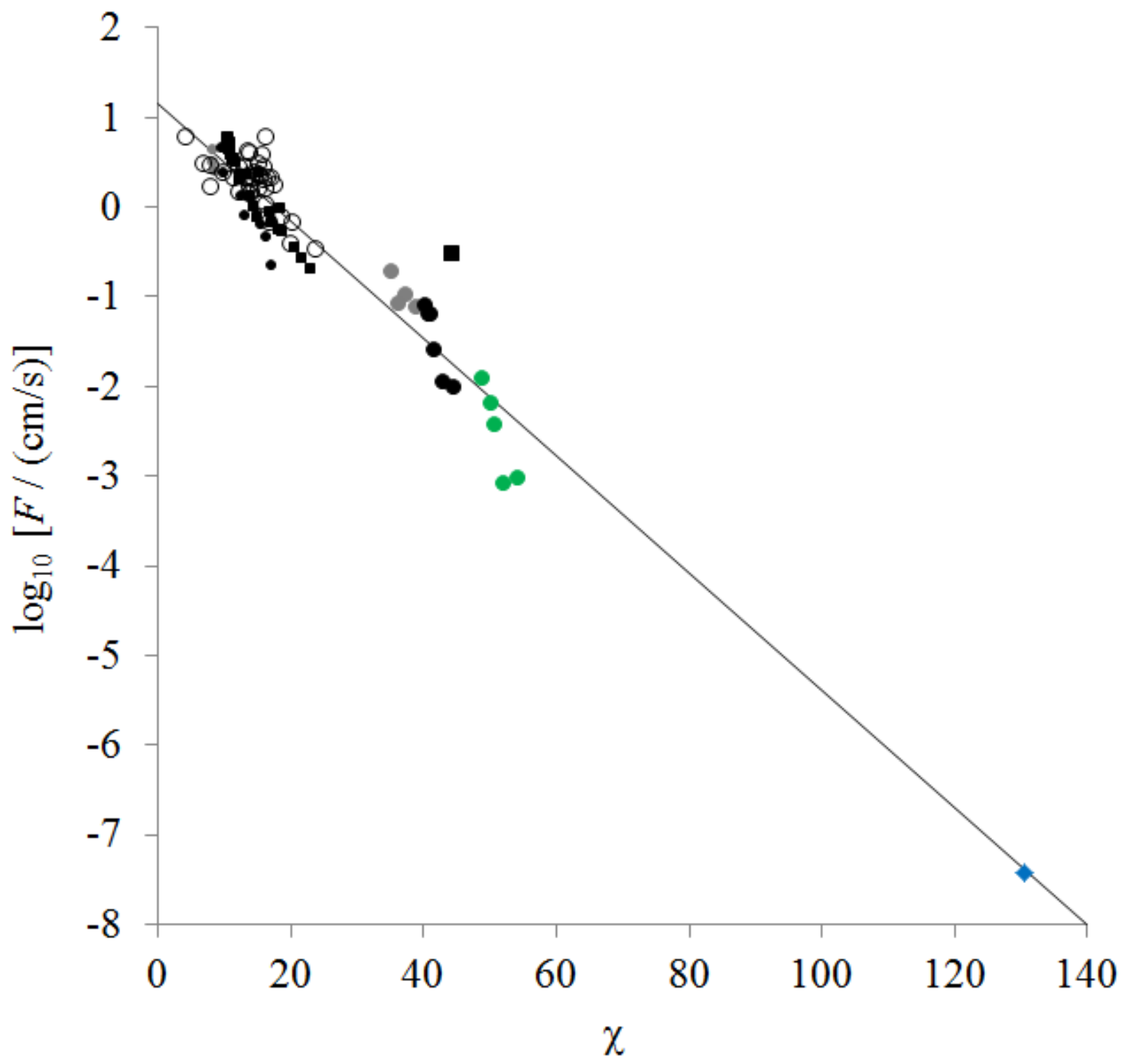




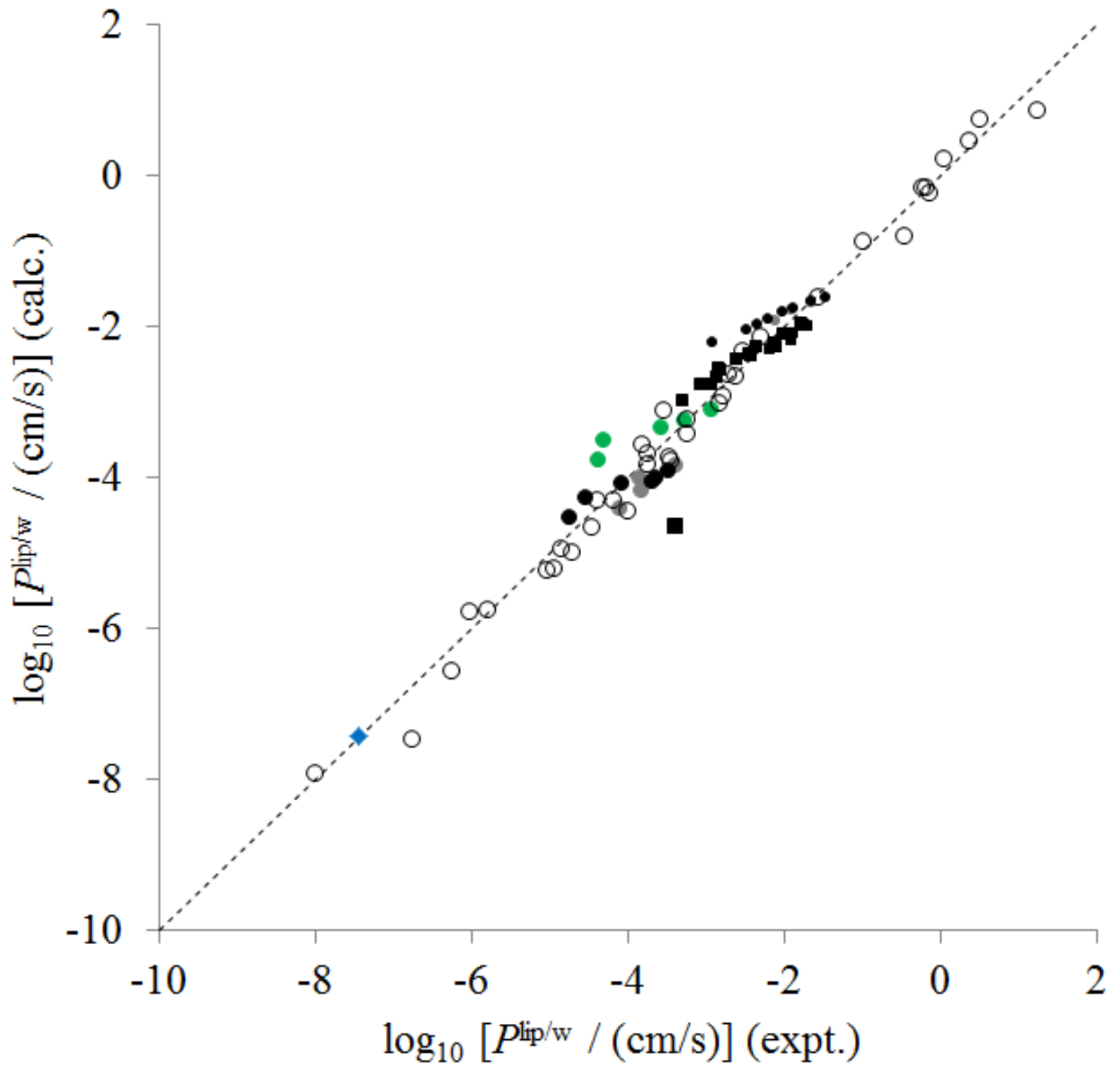


Table 1. Permeability coefficients $P^{\mathrm{lip} / \mathrm{w}}$ reported by: (2) Xiang and Anderson ${ }^{13,16}$ for acetic acid in fluid- and gel-phase DMPC and DPPC at various temperatures $T$ and cholesterol mole fractions $X_{\text {chol }}$; (3) Xiang and Anderson ${ }^{23}$ for formic acid, acetic acid and butyric acid in fluidand gel-phase pure DPPC at various temperatures $T$; and (4) Joguparthi et al. ${ }^{26}$ for DB-67 lactone in gel-phase pure DSPC at $37{ }^{\circ} \mathrm{C}$. (Numbers within parentheses correspond to the identification of data sets in the text.) Experimental results are listed as mean value \pm standard deviation; standard deviations cannot be given for data points read from graphs. The normalized surface density $\sigma^{\prime}$ is included for subsequent data analysis. Formic acid has MW $=46.025$ and $\left.K^{\text {decadiene } / \mathrm{w}}\right|_{25^{\circ} \mathrm{C}}=(6.2 \pm 0.4) \times 10^{-4} ;$ acetic acid has MW $=60.052$ and $\left.K^{\text {decadiene } / \mathrm{w}}\right|_{25^{\circ} \mathrm{C}}=(1.3 \pm$ $0.2) \times 10^{-3}$; butyric acid has MW $=88.105$ and $\left.K^{\text {decadiene } / \mathrm{w}}\right|_{25^{\circ} \mathrm{C}}=(3.9 \pm 0.1) \times 10^{-2}\left(\right.$ see Part I ${ }^{7}$ Table 1 and references cited therein). DB-67 lactone has MW $=478.612$ and $K^{\text {decadiene } / \mathrm{w}}=1.08$ $\pm 0.11 .^{26}$ (This last partition coefficient was measured at $37^{\circ} \mathrm{C}$; it is used without any temperature correction in the absence of data on the transfer enthalpy. ${ }^{20}$ )

\begin{tabular}{|c|c|c|c|c|c|c|}
\hline Solute & Lipid & $T\left({ }^{\circ} \mathrm{C}\right)$ & $X_{\text {chol }}$ & Phase & $\sigma^{\prime a}$ & $P^{\text {lip/w }}(\mathrm{cm} / \mathrm{s})$ \\
\hline \multirow[t]{17}{*}{ Acetic acid } & \multirow[t]{17}{*}{ DMPC } & 20 & \multirow[t]{5}{*}{0.00} & Gel & 0.8458 & $(3.9 \pm 0.5) \times 10^{-4 c}$ \\
\hline & & 26 & & \multirow[t]{4}{*}{ Fluid } & 0.5787 & $(6.5 \pm 0.6) \times 10^{-3 b, c}$ \\
\hline & & 30 & & & 0.5717 & $(1.2 \pm 0.4) \times 10^{-2 b, c}$ \\
\hline & & 33 & & & 0.5666 & $(1.3 \pm 0.3) \times 10^{-2 b, c}$ \\
\hline & & 36 & & & 0.5614 & $(2.0 \pm 0.4) \times 10^{-2 b, c}$ \\
\hline & & 26 & \multirow[t]{3}{*}{0.10} & \multirow[t]{3}{*}{ Fluid } & 0.6013 & $(3.6 \pm 0.3) \times 10^{-3 b}$ \\
\hline & & 30 & & & 0.5940 & $(7.5 \pm 0.4) \times 10^{-3 b}$ \\
\hline & & 40 & & & 0.5763 & $(1.6 \pm 0.3) \times 10^{-2 b}$ \\
\hline & & 26 & \multirow[t]{4}{*}{0.20} & \multirow[t]{4}{*}{ Fluid } & 0.6487 & $(1.4 \pm 0.1) \times 10^{-3 b, c}$ \\
\hline & & 30 & & & 0.6409 & $(2.4 \pm 0.2) \times 10^{-3 b, c}$ \\
\hline & & 35 & & & 0.6313 & $(4.2 \pm 0.4) \times 10^{-3 b, c}$ \\
\hline & & 40 & & & 0.6218 & $(9.4 \pm 1.0) \times 10^{-3 b, c}$ \\
\hline & & 28 & \multirow[t]{5}{*}{0.30} & \multirow[t]{5}{*}{ Fluid } & 0.6983 & $(1.1 \pm 0.1) \times 10^{-3 b}$ \\
\hline & & 30 & & & 0.6941 & $(1.3 \pm 0.1) \times 10^{-3 a}$ \\
\hline & & 37 & & & 0.6795 & $(2.4 \pm 0.2) \times 10^{-3 b}$ \\
\hline & & 39 & & & 0.6754 & $(3.5 \pm 0.3) \times 10^{-3 b}$ \\
\hline & & 50 & & & 0.6532 & $(1.7 \pm 0.3) \times 10^{-2 b}$ \\
\hline
\end{tabular}




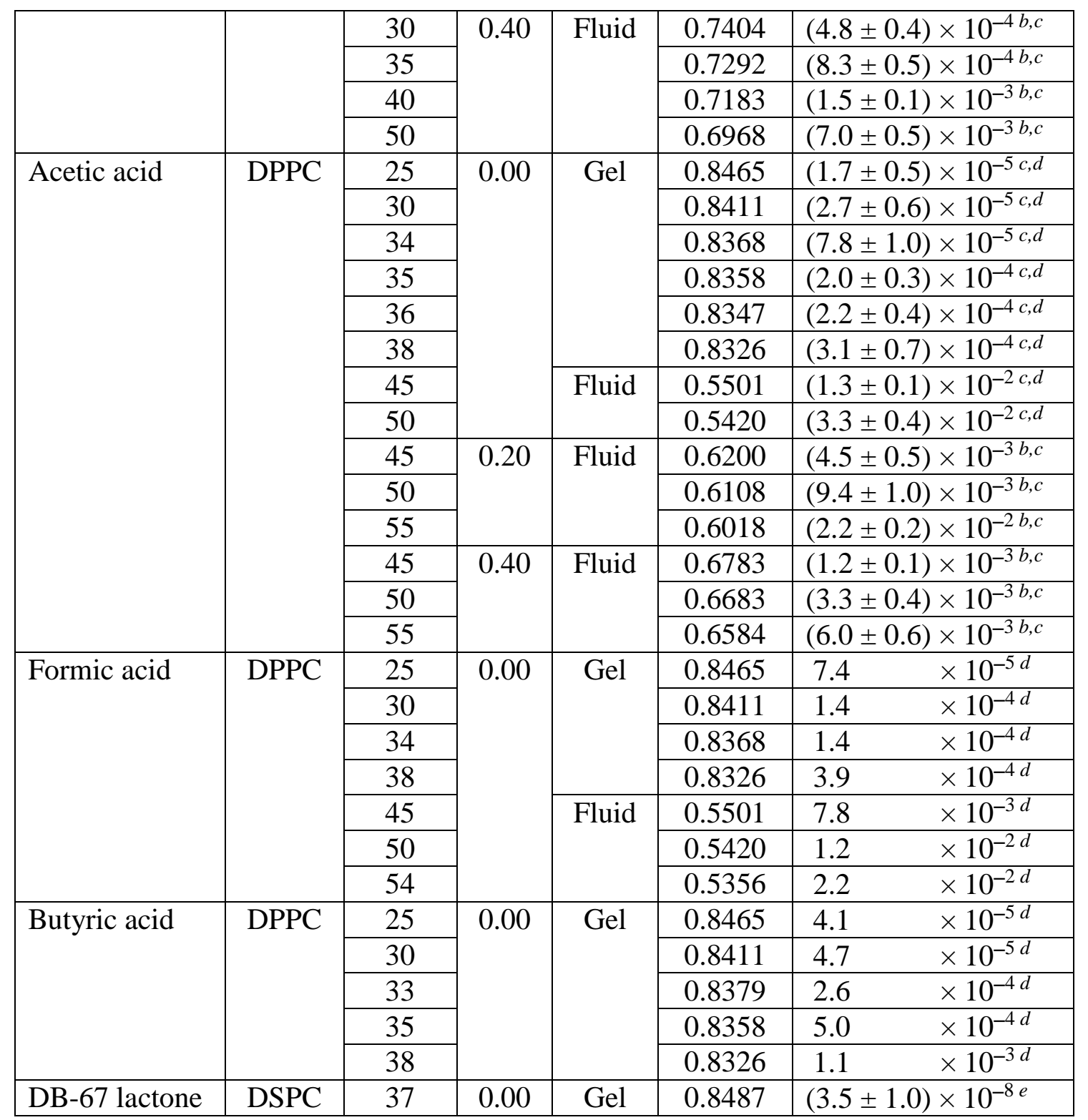

${ }^{a}$ Listed values of $\sigma^{\prime}$ are calculated according to Eq. (5) using the final value of the hard-core area per chain $A_{\mathrm{c}, 0}=17.1 \AA^{2}$ determined in the Results section below.

${ }^{b}$ Xiang and Anderson (1995). ${ }^{13}$

${ }^{c}$ Xiang and Anderson (1997). ${ }^{16}$

${ }^{d}$ Xiang and Anderson (1998). ${ }^{23}$

${ }^{e}$ Joguparthi et al. (2008). ${ }^{26}$ 
Table 2. Formulas used to calculate the area per phospholipid $A$ in the fluid phase, and the area per chain $A_{\mathrm{C}}$ in the gel phase, for pure phosphatidylcholines, based on data reported in the lipid biophysics literature. ${ }^{33,34,38-41}$ The symbol $\alpha_{A}$ denotes the coefficient of thermal area expansion (applicable to $A$ ), and $\theta$ denotes the tilt angle of fatty acid chains in the gel phase.

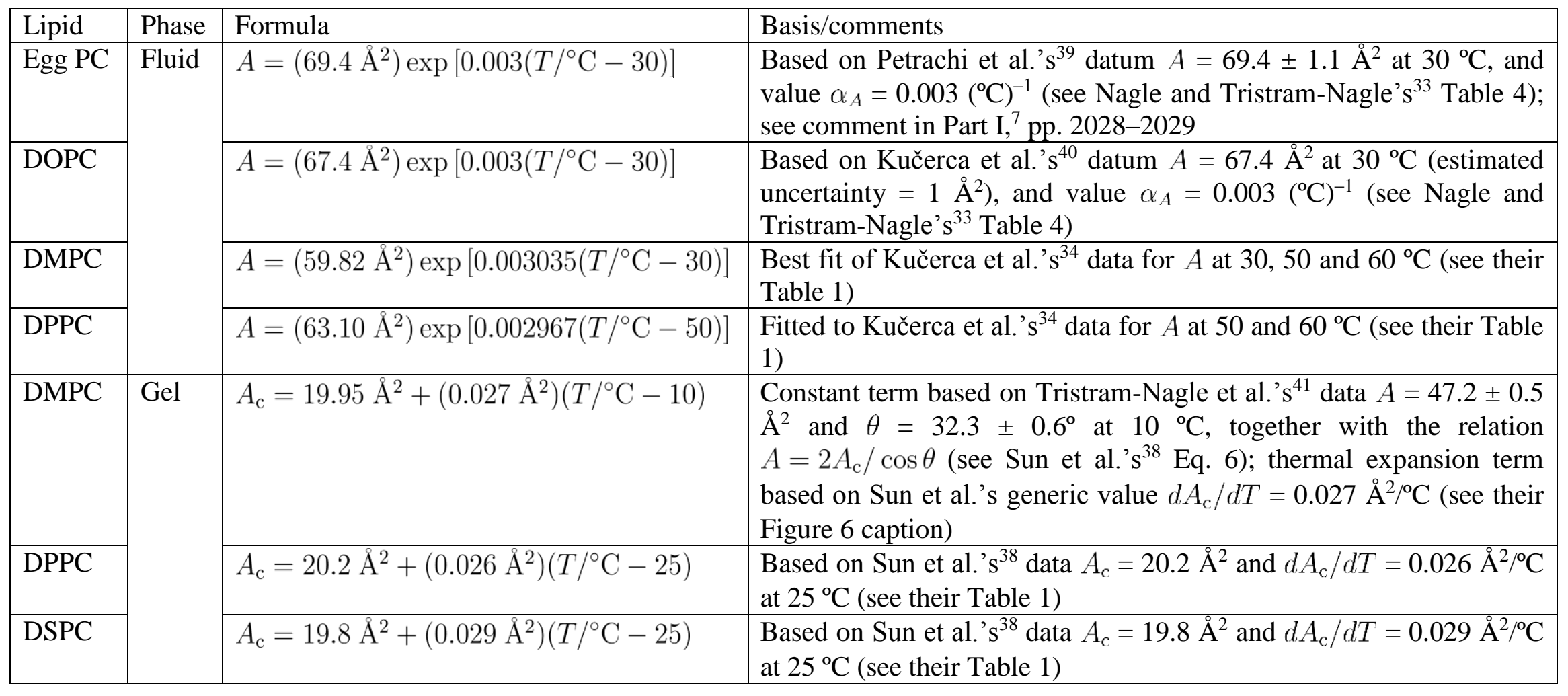


Table 3. Available values for the cholesterol correction factor $f_{\text {chol }}$ defined by Eq. 6. Division of the pure-phospholipid area $A$ by this factor yields the partial specific area for the phospholipid species in the mixture, i.e., $A\left(\right.$ lip $\left., T, X_{\text {chol }}\right)=A($ lip $, T, 0) / f_{\text {chol }}\left(X_{\text {chol }}\right)$; as written this equation acknowledges the necessity of assuming a universal (temperature-independent) dependence of $f_{\text {chol }}$ upon $X_{\text {chol }}$.

\begin{tabular}{|l|c|c|}
\hline Lipid & $X_{\text {chol }}$ & $f_{\text {chol }}$ \\
\hline DMPC $^{a}$ & 0.00 & 1.000 \\
\cline { 2 - 3 } & 0.10 & 1.039 \\
\cline { 2 - 3 } & 0.20 & 1.121 \\
\cline { 2 - 3 } & 0.30 & 1.214 \\
\cline { 2 - 3 } & 0.40 & 1.295 \\
\hline DPPC $^{b}$ & 0.00 & 1.000 \\
\cline { 2 - 3 } & 0.10 & 1.045 \\
\cline { 2 - 3 } & 0.20 & 1.127 \\
\cline { 2 - 3 } & 0.30 & 1.190 \\
\cline { 2 - 3 } & 0.40 & 1.233 \\
\hline
\end{tabular}

${ }^{a}$ Read from Pan et al.'s ${ }^{43}$ Figure 5; determined at $30^{\circ} \mathrm{C}$.

${ }^{b}$ Read from Edholm and Nagle's ${ }^{45}$ Figure 4 (see also Armstrong et al.' $\mathrm{s}^{46}$ Figure 4); determined at $50^{\circ} \mathrm{C}$. 
Table 4. Summary of formulas giving the permeability coefficient $P^{\text {lip/w }}$ for any solute in a phospholipid bilayer.

\begin{tabular}{|c|c|}
\hline Quantity & Equation \\
\hline $\begin{array}{l}1,9- \\
\text { decadiene/w } \\
\text { ater partition } \\
\text { coefficient }\end{array}$ & $\begin{array}{l}\text { In the absence of an experimental value, this coefficient can be estimated using } \\
\text { the equation }{ }^{20} \\
\left.\log _{10} K^{\text {decadiene } / \mathrm{w}}\right|_{25^{\circ} \mathrm{C}}=0.2842+1.1129 \log _{10} K^{\text {oct } / \mathrm{w}}-3.5643 A-1.1091 S-0.60 \\
\text { in which } K^{\text {oct } / \mathrm{w}} \text { denotes the octanol/water partition coefficient of the solute, and } \\
A, S, E \text { and } V \text { denote four of its Abraham solvation parameters }{ }^{10,21,22}\end{array}$ \\
\hline $\begin{array}{l}\text { Area per } \\
\text { phospholipid } \\
\text { and area per } \\
\text { fatty acid } \\
\text { chain }\end{array}$ & $\begin{array}{l}\text { Pure phospholipid bilayers: Table } 2 \text { gives formulas to compute area per } \\
\text { phospholipid } A \text { and area per chain } A_{\mathrm{c}} \text { for various phospholipids } \\
\text { Phospholipid bilayers with cholesterol: } A \text { is the partial specific area of the } \\
\text { phospholipid, and is given by } A=A_{\text {pure phospholipid }} / f_{\text {chol }}\left(X_{\text {chol }}\right) \text { with values of } \\
f_{\text {chol }}\left(X_{\text {chol }}\right) \text { drawn from Table } 3\end{array}$ \\
\hline $\begin{array}{l}\text { Relative } \\
\text { surface } \\
\text { density of } \\
\text { lipid }\end{array}$ & $\sigma^{\prime}=\left\{\begin{array}{lll}\left(17.1 \AA^{2}\right) /(A / 2), & T>T_{\mathrm{m}} & \text { (fluid phase) } \\
\left(17.1 \AA^{2}\right) / A_{\mathrm{c}}, & T<T_{\mathrm{m}} & \text { (gel phase) }\end{array}\right.$ \\
\hline $\begin{array}{l}\text { Solvent } \\
\text { factor }\end{array}$ & $\begin{array}{l}f_{\text {solvent }}=\left(\frac{100}{\mathrm{MW}}\right)^{0.56} \times \exp \left[-5.5 \times 10^{3} \mathrm{~K}\left(\frac{1}{T}-\frac{1}{298.15 \mathrm{~K}}\right)\right] \\
\text { with } T \text { in degrees Kelvin }(\mathrm{K})\end{array}$ \\
\hline $\begin{array}{l}\text { Correlating } \\
\text { variable }\end{array}$ & $\chi=\frac{(\mathrm{MW})^{0.51} \sigma^{\prime}}{1-\sigma^{\prime}}$ \\
\hline $\begin{array}{l}\text { Normalized } \\
\text { permeability }\end{array}$ & $F(\chi)=(14.4 \mathrm{~cm} / \mathrm{s}) \exp (-0.151 \chi)$ \\
\hline $\begin{array}{l}\text { Dimensional } \\
\text { permeability } \\
\text { coefficient }\end{array}$ & $P^{\text {lip } / \mathrm{w}}=\left.K^{\text {decadiene } / \mathrm{w}}\right|_{25^{\circ} \mathrm{C}} f_{\text {solvent }} F(\chi)$ \\
\hline
\end{tabular}

